\title{
SUSPENSÕES DE CIRURGIAS ORTOPÉDICAS POR MOTIVOS EXTRÍNSECOS EM PACIENTES IDOSOS
}

\author{
Suspension of orthopedic surgeries for extrinsic reasons in older patients
}

\author{
Suspensiones para cirugía ortopédica por razones extrínsecas en pacientes ancianos \\ Milena Bizo ${ }^{1}$, Maria Clara Vioto Gragnani ${ }^{2}$ (D), Thainá Oliveira Laluce ${ }^{3 *}$ (D), Jaqueline Lopes Gouveia ${ }^{4}$ (D), \\ Priscila Buck de Oliveira Ruiz ${ }^{5}$ (D), Rita Cássia Helú Mendonça Ribeiro 6 (1)
}

RESUMO: Objetivo: Verificar taxa e motivos extrínsecos de suspensões de cirurgias ortopédicas eletivas em idosos e o percurso até sua realização, reconhecendo sexo biológico, faixa etária, procedência e tipo de cirurgia. Método: Estudo retrospectivo, com delineamento descritivo, abordagem quantitativa do tipo analítica, com correlação entre variáveis, realizado em um hospital de ensino no período de janeiro a junho do ano de 2018 com idosos de 60 anos e acima, pacientes de cirurgias ortopédicas. Resultados: Das 543 cirurgias ortopédicas de idosos agendadas, 93 (17,41\%) foram suspensas. O maior motivo para a suspensão foi extrapolação do tempo da cirurgia anterior (35,48\%); a cirurgia foi cancelada uma vez e realizada entre um e dois dias após a suspensão $(91,87 \%)$, com significância estatística $(\mathrm{p}=0,00)$. Conclusão: Constatou-se que a taxa de suspensão cirúrgica nos idosos ortopédicos, por motivos extrínsecos, foi de 17,41\%. A suspensão cirúrgica ainda é um grande desafio aos profissionais e às instituições de saúde, demonstrando grande envolvimento do enfermeiro em detectar e solucionar o atraso cirúrgico, de modo a diminuir sua incidência.

Palavras-chave: Idoso. Ortopedia. Procedimentos cirúrgicos eletivos. Gestão em saúde. Enfermagem perioperatória.

ABSTRACT: Objective: To identify the rates and extrinsic reasons for the suspension of elective orthopedic surgeries in older adults and the course until their performance, according to sex, age group, origin, and type of surgery. Method: This is a retrospective descriptive study with a quantitative analytical approach and variable correlation, carried out in a teaching hospital from January to June 2018. The sample consisted of orthopedic surgery patients aged 60 years and older. Results: Out of the 543 orthopedic surgeries scheduled for older adults, 93 (17.41\%) were suspended. The main reason for suspension was the previous surgery lasting longer than expected (35.48\%); the surgery was canceled once and performed 1-2 days after the suspension (91.87\%), with statistical significance $(\mathrm{p}=0.00)$. Conclusion: We found that the rate of surgical suspension for extrinsic reasons in orthopedic older patients was $17.41 \%$. Surgical suspension remains a great challenge for professionals and health facilities, showing the significant involvement of nurses in detecting and solving surgical delays in order to decrease their incidence.

Keywords: Aged. Orthopedics. Elective surgical procedures. Health management. Perioperative nursing.

RESUMEN: Objetivo: Verificar la tasa y motivos extrínsecos de las suspensiones electivas de cirugía ortopédica en ancianos y la vía para su realización, reconociendo el sexo biológico, grupo de edad, origen y tipo de cirugía. Método: estudio retrospectivo, con diseño descriptivo, abordaje analítico cuantitativo, con correlación entre variables, realizado en un hospital de enseñanza, de enero a junio del año 2018, con ancianos de 60 años y más, sometidos a cirugía ortopédica. Resultados: De las 543 cirugías ortopédicas programadas para ancianos, $93(17,41 \%)$ fueron suspendidas. El mayor motivo de suspensión fue la extrapolación del momento de la cirugía anterior $(35,48 \%)$; la cirugía se canceló una vez y se realizó entre uno y dos días después de la

\footnotetext{
'Enfermeira, pós-graduanda do Programa de Residência Multiprofissional de Saúde do Adulto e do Idoso, Faculdade de Medicina de Botucatu, Universidade Estadual Paulista “Júlio de Mesquita Filho" (UNESP) - Botucatu, (SP), Brasil.

${ }^{2}$ Bacharela em Enfermagem pela Faculdade de Medicina de São José do Rio Preto (FAMERP). Enfermeira em Unidade de Pronto Atendimento do Hospital de Caridade São Vicente de Paulo - Jundiaí (SP), Brasil. ${ }^{3}$ Enfermeira, pós-graduanda do Programa de Residência Uniprofissional em Centro Cirúrgico e Central de Material e Esterilização, Hospital Sírio-Libânes - São Paulo (SP), Brasil. «Especialista em Centro Cirúrgico, Recuperação Anestésica e Central de Materiais e Esterilização pela FAMERP. Enfermeira no Centro Cirúrgico do Hospital de Base de São José do Rio Preto - São José do Rio Preto (SP), Brasil.

${ }^{5}$ Enfermeira, mestre pelo Programa de Pós-Graduação de Enfermagem da FAMERP - São José do Rio Preto (SP), Brasil.

${ }^{6}$ Doutora em Ciências e Saúde pela FAMERP. Professora da FAMERP - São José do Rio Preto (SP), Brasil.

*Autora correspondente: thainalaluce@gmail.com

Recebido: 20/09/2020 - Aprovado: 23/03/2021

https://doi.org/10.5327/Z1414-4425202100020003
} 
suspensión $(91,87 \%)$, con significación estadística $(\mathrm{p}=0,00)$. Conclusión: Se encontró que la tasa de suspensión quirúrgica, en el anciano ortopédico por razones extrínsecas, fue del 17,41\%. La suspensión quirúrgica sigue siendo un gran desafío para los profesionales y las instituciones de salud, demostrando la gran implicación del enfermero en la detección y resolución del retraso quirúrgico, con el fin de reducir su incidencia.

Palablas clave: Anciano. Ortopedia. Procedimientos quirúrgicos electivos. Gestión en salud. Enfermería perioperatoria.

\section{INTRODUÇÃ̃o}

Nas últimas décadas, houve um declínio da taxa de fecundidade da população mundial, além do aumento da expectativa de vida. Essa mudança na pirâmide etária traz maiores problemas de saúde pública, pois afeta um grupo de vulnerabilidade, os idosos ${ }^{1}$. Segundo a Organização Mundial da Saúde (OMS), nos países em desenvolvimento, como o Brasil, idoso é o indivíduo que tem 60 anos ou mais; nos países desenvolvidos esse número aumenta para 65 anos ${ }^{1}$.

Com o avanço da idade, as internações também aumentam e diversas modificações funcionais e estruturais ocorrem, como diminuição da massa corpórea, perda natural de densidade óssea, doenças degenerativas, dificuldades auditivas e oftalmológicas ${ }^{2,3}$. Uma das maiores causas de hospitalização de idosos é a queda, caracterizada por um evento involuntário que resulta em mudança da posição inicial para o mesmo nível ou nível mais baixo, provocando uma ou mais lesões². Os idosos são o grupo mais acometido por esse acidente e sua recorrência é elevada, sendo fator importante para o desenvolvimento de complicações crônicas ${ }^{2}$.

Algumas modificações decorrentes da idade interferem diretamente no processo cirúrgico, como a diminuição da massa magra e da água total, que são fatores predisponentes de maior toxicidade por fármacos e maior risco de hipotermia ${ }^{3}$. As alterações fisiológicas naturais, associadas às comorbidades, levam à predisposição para quedas, que são as maiores causas de fraturas ósseas e, por vezes, são tratadas cirurgicamente ${ }^{4}$. Conhecendo a vulnerabilidade desse grupo, o procedimento cirúrgico causa maior preocupação na equipe multiprofissional, e faz-se necessário maior cautela no período perioperátório ${ }^{3}$.

Os procedimentos cirúrgicos envolvem diversos fatores, que podem ser intrínsecos, relacionados ao paciente, ou extrínsecos, relacionados ao ambiente e à equipe $\mathrm{e}^{4}$. Desordens nesses fatores podem gerar suspensão e postergação da cirurgia. Isso traz consequências para o paciente, que não ficará isento desse ato invasivo, mas permanecerá hospitalizado e exposto a agentes infecciosos, muitas vezes restrito ao seu leito e sujeito a atrofia muscular, lesões por pressão, osteopenia, pneumonia, tromboembolismo pulmonar e embolização gordurosa ${ }^{5,6}$. Além disso, isso gera prejuízos para a instituição pelo aumento dos custos com hospitalização, medicações, alimentação (paciente e acompanhante), insumos e profissionais ${ }^{4}$.

Segundo estudo realizado em um hospital público de ensino no estado de Minas Gerais, a taxa de suspensão das cirurgias foi de $14,14 \%$ e na maioria delas (63,78\%) não houve registro do motivo. Das suspensões justificadas, em $8,82 \%$ o paciente não se internou, a ausência de condições clínicas adequadas representou $8,30 \%$ e as questões administrativas $8,14 \%$. Entre 15 especialidades, as que mais suspenderam cirurgias foram urologia e ortopedia ${ }^{7}$.

Diante de tais constatações, justifica-se o presente estudo, com foco na suspensão de cirurgias ortopédicas na população idosa.

\section{OBJETIVO}

Identificar a taxa e os motivos extrínsecos de suspensões de cirurgias ortopédicas eletivas em idosos, bem como o percurso até a sua realização, reconhecendo sexo biológico, faixa etária, procedência e tipo de cirurgia.

\section{MÉTODO}

Estudo retrospectivo, com delineamento descritivo, abordagem quantitativa do tipo analítica, com correlação entre variáveis, realizado no período de janeiro a junho de 2018.

O cenário do estudo foi um hospital de ensino de porte especial, com capacidade extra (708 leitos), localizado na região Sudeste do Brasil. A instituição presta atendimento a 2 milhões de habitantes de 102 municípios da Divisão Regional de Saúde de Rio Preto (DRS 15), pacientes dos sistemas público e privado de saúde. No sistema público, atende aos usuários do Sistema Único de Saúde (SUS) e, no sistema privado, aos beneficiários do Sistema de Saúde Suplementar (SSS), composto de várias operadoras e seguradoras de saúde credenciadas ao hospital, além de pacientes particulares.

A pesquisa foi realizada no Centro Cirúrgico (CC), que é composto de 28 salas operatórias (SO), com média de 31.714 cirurgias/ano, majoritariamente feitas pelo SUS. 
Para atendermos aos objetivos deste estudo, optamos por realizar uma análise de prontuário eletrônico dos pacientes submetidos a cirurgias eletivas ortopédicas com idade maior ou igual a 60 anos. Foram excluídos os pacientes que tiveram sua cirurgia cancelada por motivos intrínsecos. Foram coletadas informações existentes em 93 registros.

Após a tabulação dos dados coletados, exerceram-se duas funções de análises estatísticas: descritiva e inferencial. De maneira descritiva, traçou-se o perfil da amostra estudada, contemplando as variáveis analisadas e seus desdobramentos. Os dados foram replicados de forma absoluta e relativa nessa primeira parte.

No âmbito inferencial, traçou-se como objetivo estatístico a análise de independência e predição entre as variáveis propostas no escopo do trabalho. Para isso, utilizou-se, no escopo dos padrões esperados, o teste U de Mann-Whitney. Os resultados da dependência entre as variáveis propostas foram obtidos por meio de análise dos valores $\mathrm{p}$. Um valor $\mathrm{p}$ inferior a 0,05 foi considerado estatisticamente significativo.

Todas as análises foram realizadas usando-se o software Statistical Package for the Social Sciences (SPSS Statistics), versão 23, atrelado às funcionalidades da ferramenta Excel ${ }^{\circledR}$, versão 2016.

Para o cálculo da taxa de suspensão cirúrgica, utilizou-se a fórmula do compromisso com a qualidade hospitalar $(\mathrm{CQH})$, que compreende o número de cirurgias suspensas por fatores extrapaciente multiplicado por 100 e dividido pelo total de cirurgias agendadas ${ }^{8,9}$.

Este estudo respeitou os trâmites éticos que regem as pesquisas com seres humanos, estabelecidos pela Resolução $n^{\circ}$ 466/2012 do Conselho Nacional de Saúde. Foi submetido ao Comitê de Ética em Pesquisa (CEP) da Faculdade de Medicina de São José do Rio Preto (FAMERP), aprovado como ementa com o parecer $\mathrm{n}^{\circ} 2.748 .090$, em 2 de março de 2018, sendo parte do projeto mãe "Estudo da Qualidade de Vida de Idosos" CEP-FAMERP, que tem parecer n ${ }^{\circ}$ 1.508.014.

\section{RESULTADOS}

No recorte temporal analisado, de janeiro a junho de 2018, o CC teve 11.511 cirurgias agendadas, considerando-se todas as especialidades com que o hospital opera. Delas, 1.117 (9,70\%) eram cirurgias ortopédicas, sendo $543(48,61 \%)$ em pacientes idosos. Nos meses da pesquisa o total de cancelamentos foi de 1.185 procedimentos, 394 (33,24\%) de cirurgias ortopédicas e 125 $(31,72 \%)$ em pacientes idosos. Levando-se em conta os motivos extrínsecos e intrínsecos do cancelamento, no entanto, 93 (74,4\%) foi o número de idosos que tiveram sua cirurgia cancelada por motivos extrínsecos, ou seja, por motivos do próprio CC do hospital. Aplicando a fórmula do $\mathrm{CQH}$ nos dados coletados, verificou-se que a taxa de cirurgias suspensas foi de $17,41 \%$.

$\mathrm{Na}$ análise descritiva da amostra, das 93 suspensões cirúrgicas dos idosos, 22 (23,66\%) ocorreram no mês de março, 67 $(72,04 \%)$ em mulheres, $63(67,74 \%)$ em pacientes procedentes de outros municípios, 38 (40,86\%) naqueles com idade entre 60 e 69 anos, 55 (59,14\%) ocorreram uma vez, $24(25,80 \%)$ tiveram tempo de espera de um a dois dias, e $33(35,48 \%)$ foram motivadas pelo fato de a cirurgia anterior ter ultrapassado o tempo previsto. Ainda, $29(31,18 \%)$ cirurgias foram decorrentes de fratura do fêmur e $11(11,83 \%)$ de fratura de tíbia.

O estudo encontrou correlação estatisticamente significante na análise estatística inferencial dos cruzamentos de faixa etária $\times$ tempo de espera para cirurgia $(\mathrm{p}=0,004)$; vezes de suspensão $\times$ tempo de espera pela cirurgia $(\mathrm{p}=0,000)$; vezes de suspensão $\times$ motivo de suspensão $(\mathrm{p}=0,015)$; mês $\times$ motivo de suspensão $(\mathrm{p}=0,014)$ e mês $\times$ tipo de cirurgia $(\mathrm{p}=0,027)$. Foram feitos testes de hipóteses com o método $\mathrm{U}$ de Mann-Whitney, analisando-se o comportamento das correlações entre as variáveis analisadas e o grau de explicação da variável dependente em relação às independentes da amostra.

No cruzamento mês $\times$ tipo de cirurgia, foram encontrados 19 tipos de cirurgias, sendo a de maior frequência a decorrente de fratura de fêmur (29/31,18\%), seguida de fratura de tíbia (11/11,83\%), e as demais tiveram índice menor de $10 \%(\mathrm{p}=0,027)$.

Com relação à correlação da faixa etária com o tempo de espera para a cirurgia, houve evidencia estatística; idosos de 60 a 69 anos, em sua maioria, esperavam de um a dois dias para realizar a cirurgia, mas grande parte deles esperou mais de 30 dias (87,50\%), seguidos dos idosos de 80 a 89 anos, que esperaram de 21 a 30 dias (66,67\%), quando comparados com as demais idades $(\mathrm{p}=0,004)$, conforme demonstrado na Tabela 1 .

Outro fator significante foi o tempo de espera e a quantidade de vezes em que ocorreu a suspensão $(\mathrm{p}=0,00)$. O total de $91,67 \%$ cirurgias foi suspenso uma vez e realizado entre um e dois dias depois, e $88,24 \%$ foram efetivadas entre três e cinco dias depois; as demais esperaram um tempo de até mais de 30 dias para serem realizadas (Tabela 2).

Entre os maiores motivos de suspensão cirúrgica, está a extrapolação do tempo previsto da cirurgia anterior $(35,48 \%)$, que se correlaciona com os meses pesquisados $(\mathrm{p}=0,014)$, a exemplo de janeiro, com 69,23\% suspensões (Tabela 3 ). Percentagem significativa das cirurgias (50\%) foi cancelada duas vezes, havendo correlação com o motivo do cancelamento ( $\mathrm{p}=0,014)$, conforme demonstrado na Tabela 4 . 
Tabela 1. Tempo de espera para cirurgia segundo faixa etária dos pacientes.

\begin{tabular}{|c|c|c|c|c|c|c|c|c|c|c|c|c|c|c|c|c|c|c|}
\hline \multirow{2}{*}{$\begin{array}{l}\text { Faixa } \\
\text { Etária } \\
\text { (anos) } \\
p=0,004^{*}\end{array}$} & \multicolumn{2}{|c|}{$\begin{array}{c}\text { Não } \\
\text { Realizadas }\end{array}$} & \multicolumn{2}{|c|}{$\begin{array}{l}1 \text { a } 2 \\
\text { dias }\end{array}$} & \multicolumn{2}{|c|}{$\begin{array}{r}3 \text { a } 5 \\
\text { dias }\end{array}$} & \multicolumn{2}{|c|}{$\begin{array}{r}6 \text { a } 8 \\
\text { dias }\end{array}$} & \multicolumn{2}{|c|}{$\begin{array}{l}9 \text { a } 10 \\
\text { dias }\end{array}$} & \multicolumn{2}{|c|}{$\begin{array}{l}11 \text { a } 20 \\
\text { dias }\end{array}$} & \multicolumn{2}{|c|}{$\begin{array}{l}21 \text { a } 30 \\
\text { dias }\end{array}$} & \multicolumn{2}{|c|}{$\begin{array}{l}>30 \\
\text { dias }\end{array}$} & \multicolumn{2}{|c|}{ Total } \\
\hline & $\mathbf{N}$ & $\%$ & $\mathbf{N}$ & $\%$ & $\mathbf{N}$ & $\%$ & $\mathbf{N}$ & $\%$ & $\mathbf{N}$ & $\%$ & $\mathbf{N}$ & $\%$ & $\mathbf{N}$ & $\%$ & $\mathbf{N}$ & $\%$ & $\mathbf{N}$ & $\%$ \\
\hline 60 a 69 & 3 & 21,43 & 10 & 41,67 & 8 & 47,06 & 7 & 36,84 & 1 & 50 & 2 & 33,33 & - & - & 7 & 87,50 & 38 & 40,86 \\
\hline 70 a 79 & 4 & 28,57 & 10 & 41,67 & 6 & 35,29 & 5 & 26,32 & - & - & 2 & 33,33 & 1 & 33,33 & 1 & 12,50 & 29 & 31,18 \\
\hline 80 a 89 & 5 & 35,71 & 3 & 12,50 & 2 & 11,76 & 7 & 36,84 & 1 & 50 & 2 & 33,33 & 2 & 66,67 & - & - & 22 & 23,66 \\
\hline$>90$ & 2 & 14,29 & 1 & 4,17 & 1 & 5,88 & - & - & - & - & - & - & - & - & - & - & 4 & 4,30 \\
\hline Total & 14 & 100 & 24 & 100 & 17 & 100 & 19 & 100 & 2 & 100 & 6 & 100 & 3 & 100 & 8 & 100 & 93 & 100 \\
\hline
\end{tabular}

*Valor significativo; Método U de Mann-Whitney.

Tabela 2. Tempo de espera para cirurgia, de acordo com o número de vezes em que o procedimento foi suspenso.

\begin{tabular}{|c|c|c|c|c|c|c|c|c|c|c|c|c|c|c|c|c|c|c|}
\hline \multirow{2}{*}{$\begin{array}{l}\text { Vezes de } \\
\text { Suspensão } \\
p=0,000^{*}\end{array}$} & \multicolumn{2}{|c|}{$\begin{array}{c}\text { Não } \\
\text { Realizou }\end{array}$} & \multicolumn{2}{|c|}{$\begin{array}{l}1 \text { a } 2 \\
\text { dias }\end{array}$} & \multicolumn{2}{|c|}{$\begin{array}{l}3 \text { a } 5 \\
\text { dias }\end{array}$} & \multicolumn{2}{|c|}{$\begin{array}{l}6 \text { a } 8 \\
\text { dias }\end{array}$} & \multicolumn{2}{|c|}{$\begin{array}{l}9 \text { a } 10 \\
\text { dias }\end{array}$} & \multicolumn{2}{|c|}{$\begin{array}{c}11 \text { a } 20 \\
\text { dias }\end{array}$} & \multicolumn{2}{|c|}{$\begin{array}{c}21 \text { a } 30 \\
\text { dias }\end{array}$} & \multicolumn{2}{|c|}{$\begin{array}{l}>30 \\
\text { dias }\end{array}$} & \multicolumn{2}{|c|}{ Total } \\
\hline & $\mathbf{N}$ & $\%$ & $\mathbf{N}$ & $\%$ & $\mathbf{N}$ & $\%$ & $\mathbf{N}$ & $\%$ & $\mathbf{N}$ & $\%$ & $\mathbf{N}$ & $\%$ & $\mathbf{N}$ & $\%$ & $\mathbf{N}$ & $\%$ & $\mathbf{N}$ & $\%$ \\
\hline Não realizada & 14 & 100 & - & - & - & - & - & - & - & - & - & - & - & - & - & - & 14 & 15,05 \\
\hline 1 & - & - & 22 & 91,67 & 15 & 88,24 & 7 & 36,84 & 1 & 50 & 4 & 66,67 & 2 & 66,67 & 4 & 50 & 55 & 59,14 \\
\hline 2 & - & - & 2 & 8,33 & 1 & 5,88 & 9 & 47,37 & 1 & 50 & 1 & 16,67 & - & - & 4 & 50 & 18 & 19,35 \\
\hline 3 & - & - & - & - & 1 & 5,88 & 3 & 15,79 & - & - & 1 & 16,67 & 1 & 33,33 & - & - & 06 & 6,45 \\
\hline Total & 14 & 100 & 24 & 100 & 17 & 100 & 19 & 100 & 2 & 100 & 6 & 100 & 3 & 100 & 8 & 100 & 93 & 100 \\
\hline
\end{tabular}

*Valor significativo; Método U de Mann-Whitney

Tabela 3. Motivos de suspensão das cirurgias de acordo com o mês.

\begin{tabular}{|c|c|c|c|c|c|c|c|c|c|c|c|c|c|c|}
\hline Motivo da Suspensão & \multicolumn{2}{|c|}{ Janeiro } & \multicolumn{2}{|c|}{ Fevereiro } & \multicolumn{2}{|c|}{ Março } & \multicolumn{2}{|c|}{ Abril } & \multicolumn{2}{|c|}{ Maio } & \multicolumn{2}{|c|}{ Junho } & \multicolumn{2}{|c|}{ Total } \\
\hline$p=(0,014)^{*}$ & $\mathbf{N}$ & $\%$ & $\mathbf{N}$ & $\%$ & $\mathbf{N}$ & $\%$ & $\mathbf{N}$ & $\%$ & $\mathbf{N}$ & $\%$ & $\mathbf{N}$ & $\%$ & $\mathbf{N}$ & $\%$ \\
\hline $\begin{array}{l}\text { Cirurgia anterior ultrapassou o } \\
\text { tempo previsto }\end{array}$ & 9 & 69,23 & 3 & 42,86 & 8 & 36,36 & 4 & 28,57 & 5 & 29,41 & 4 & 20 & 33 & 35,48 \\
\hline $\begin{array}{l}\text { Optou-se por operar outro } \\
\text { paciente grave/urgência }\end{array}$ & - & - & 1 & 14,29 & 2 & 9,09 & 5 & 35,71 & 4 & 23,53 & 1 & 5 & 13 & 13,98 \\
\hline $\begin{array}{l}\text { Optou-se por operar outro } \\
\text { procedimento cirúrgico }\end{array}$ & - & - & 2 & 28,57 & 3 & 13,64 & - & - & 2 & 11,76 & 9 & 45 & 16 & 17,20 \\
\hline Óbito & - & - & - & - & - & - & - & 7,14 & - & - & 1 & 5 & 2 & 2,15 \\
\hline Total & 13 & 100 & 7 & 100 & 22 & 100 & 14 & 100 & 17 & 100 & 20 & 100 & 93 & 100 \\
\hline
\end{tabular}

*Valor significativo; Método U de Mann-Whitney. Foram excluídos os motivos de suspensão com percentual menor do que 10\%. Os totais referem-se a todos os motivos de suspensões.

Tabela 4. Motivos de suspensão da cirurgia de acordo com o número de vezes em que o procedimento foi suspenso.

\begin{tabular}{|c|c|c|c|c|c|c|c|c|c|c|}
\hline \multirow{2}{*}{$\begin{array}{l}\text { Motivo da Suspensão } \\
p=(0,015)\end{array}$} & \multicolumn{2}{|c|}{$\begin{array}{c}\text { Não } \\
\text { Realizadas }\end{array}$} & \multicolumn{2}{|c|}{1 Vez } & \multicolumn{2}{|c|}{2 Vezes } & \multicolumn{2}{|c|}{3 Vezes } & \multicolumn{2}{|c|}{ Total } \\
\hline & $\mathbf{N}$ & $\%$ & $\mathbf{N}$ & $\%$ & $\mathbf{N}$ & $\%$ & $\mathbf{N}$ & $\%$ & $\mathbf{N}$ & $\%$ \\
\hline Cirurgia anterior ultrapassou o tempo previsto & - & 0 & 22 & 40 & 9 & 50 & 2 & 33,33 & 33 & 35,48 \\
\hline Encaixe de cirurgia de urgência & - & 0 & 12 & 21,82 & 1 & 5,56 & - & - & 13 & 13,98 \\
\hline Encaixe de outro procedimento cirúrgico & 2 & 14,29 & 7 & 12,73 & 5 & 27,78 & 2 & 33,33 & 16 & 17,20 \\
\hline Total & 14 & 100 & 55 & 100 & 18 & 100 & 6 & 100 & 93 & 100 \\
\hline
\end{tabular}

*Valor significativo; Método U de Mann-Whitney. Foram excluídos os motivos de suspensão com percentual menor do que 10\%. Os totais referem-se a todos os motivos de suspensões. 


\section{DISCUSSÃO}

Estudos apontam que a taxa de suspensões cirúrgicas eletivas em hospitais universitários varia entre 17 e $33 \%{ }^{10,11}$. Um estudo internacional ${ }^{11}$ constatou que a taxa de cancelamentos variou entre 1 e $23 \%$, corroborando os resultados da presente pesquisa, que encontrou taxa de 10,29\% do total de cirurgias suspensas. Em Malawi, país da África Oriental, os autores verificaram que há diferenças entre as taxas de suspensões cirúrgicas entre países subdesenvolvidos, como a África do Sul (44,5\%) e o Malawi (44,2\%), e os desenvolvidos, como o Reino Unido (0,7 a 1,1\%) e a Austrália $(7,2 \%)$, o que demonstra que o número de pessoal, o volume cirúrgico, os recursos disponíveis e a demanda são fatores predisponentes para o aumento de suspensões ${ }^{12}$.

Num estudo de análise documental de 1.449 cirurgias canceladas, a especialidade cirúrgica que apresentou maior frequência de cancelamento foi a ortopedia e, entre os principais motivos de suspensão extrínsecos ao paciente, esteve a mudança de conduta da equipe cirúrgica e a ultrapassagem de tempo cirúrgico eletivo ${ }^{13}$. O estudo também identificou que as principais causas de suspensão cirúrgica se davam por decisão do cirurgião, do anestesista e do enfermeiro, respectivamente, tendo sido avaliados tanto motivos intrínsecos como extrínsecos ao paciente ${ }^{13}$.

Em outra pesquisa, realizada no estado do Paraná ${ }^{14}$, os cancelamentos da cirurgia geral e da ortopedia ganharam destaque; a ortopedia teve taxa equivalente a $23,57 \%$, valor mais aproximado aos da nossa pesquisa.

Um estudo em um hospital escola no Paraná constatou que a taxa de suspensão de cirurgias ortopédicas foi de $26,2 \%$, sendo essa especialidade a segunda com maior taxa de suspensão, somente superada pela cirurgia torácica ${ }^{15}$. Nesse estudo, os motivos foram divididos entre os creditados ao paciente e outros. Apenas $22 \%$ das suspensões ocorreram por motivos intrínsecos, relacionados ao próprio paciente, enquanto o restante $(78 \%)$ foi creditado às subdivisões extrínsecas, o que mostra que a maior causa de suspensões relacionava-se com o próprio $\mathrm{CC}^{15}$.

Em um hospital de Belo Horizonte, capital do estado de Minas Gerais ${ }^{16}$, os autores estudaram a taxa de suspensão cirúrgica no período de julho a dezembro de 2013, incluindo motivos intrínsecos e extrínsecos ao paciente. A taxa de suspensão de cirurgias ortopédicas foi de $22,1 \%$, correspondendo ao segundo lugar. Esse dado ratifica o achado da pesquisa atual, demostrando que há mais suspensões em cirurgias ortopédicas. Entre os motivos extrínsecos de suspensão, foi observada ${ }^{16}$, principalmente, a falta de autorização do convênio (31,5\%); há um desencontro com os resultados obtidos no presente estudo, no qual obtivemos a ultrapassagem de tempo cirúrgico com maior taxa de suspensão $(35,48 \%)$. Em nossa pesquisa, constatou-se que a taxa de suspensão cirúrgica por motivos extrínsecos em pacientes idosos foi de $17,12 \%$ quando aplicada a fórmula do CQH; já um estudo no hospital de Belo Horizonte, com pacientes de todas as faixas etárias e contando com motivos intrínsecos e extrínsecos, encontrou taxa de 5,2\% no período de seis meses ${ }^{16}$.

Estudo realizado em um hospital acadêmico chinês verificou que o sexo biológico feminino apresentou diferenças significativas quando comparado com o masculino, o que tem como possível justificativa a maior procura das mulheres pelos serviços de saúde ${ }^{11}$. Esse resultado corrobora os da nossa pesquisa, visto que a maior porcentagem de suspensões cirúrgicas foi entre pacientes femininas (72,04\%).

Pesquisa conduzida em um hospital de São Paulo constatou que os pacientes idosos são os mais afetados, mostrando que as suspensões por motivos clínicos e não clínicos ocupou o segundo lugar ${ }^{17}$. Isso corrobora os achados de nosso estudo, no qual $31,72 \%$ das suspensões ocorreram em idosos por motivos intrínsecos e extrínsecos.

A respeito dos tipos de cirurgias ortopédicas com maior número de suspensão, a fratura de fêmur teve maior incidência $(31,18 \%)$ e foi preponderante em todos os meses pesquisados. Esse tipo de fratura apresenta maior ocorrência entre os idosos, em decorrências das quedas. Um estudo que avaliou a fratura de fêmur no período de 2008 a 2012 comprovou que, em consonância com outros países, o Brasil apresenta altos números de casos, de aproximadamente 36.200 ao ano, e isso se deve ao elevado número de agendamentos para essa especialidade cirúrgica ${ }^{18}$.

Em concordância com nossos resultados de ultrapassagem de tempo cirúrgico previsto $(35,48 \%)$, uma pesquisa realizada no hospital universitário da Universidade Federal de Santa Catarina (UFSC) verificou a falta de tempo hábil (24\%), seguida de falta de vagas na unidade de terapia intensiva (UTI) (12\%), como um dos principais motivos de suspensões cirúrgicas ${ }^{19}$. Em pesquisa realizada em São Paulo, os dados mostraram que os principais motivos da suspensão extrínseca ao paciente foram o avanço da hora, a falta de vaga na UTI e de equipamentos e materiais, como órteses, próteses e materiais especiais (OPME) $)^{17}$. Pode-se justificar o 
atraso no tempo por complicações ou mudanças de técnicas e condutas que ocorrem durante o procedimento cirúrgico; também a presença ou não de médicos residentes durante $o$ procedimento, bem como o porte cirúrgico a ser realizado posteriormente ${ }^{11}$.

Outra causa do atraso de cirurgias reside na falha de planejamento do mapa cirúrgico e na sua conferência, ligadas diretamente à gestão da equipe por parte do enfermeiro, bem como da comunicação assertiva entre os envolvidos e o conhecimento prévio dos materiais e equipamentos do hospital. A taxa de cancelamento de cirurgia remete ao imperativo de reduzi-la; para tal, faz-se indispensável o monitoramento contínuo desse indicador e a implementação de estratégias para sua redução. É necessária uma boa gestão de processos e estratégias para que o enfermeiro consiga detectar e solucionar o atraso cirúrgico, sendo imprescindível a atitude proativa e o empoderamento desse profissional para beneficiar o maior número de pacientes possível ${ }^{20,21}$.

O uso de ferramentas gerenciais, como matriz de gravidade, urgência e tendência (GUT) e diagrama de Ishikawa, é um potente colaborador na resolução de desafios no CC, já que o planejamento ineficaz está entre $21 \%$ das causas ${ }^{21}$.

De acordo com estudo realizado em um hospital público de referência do estado de Pernambuco, no período de fevereiro de 2015 a fevereiro de 2016, as especialidades mais propensas à suspensão foram cirurgia geral, ortopedia e urologia, nessa ordem. Entre os motivos extrínsecos ao paciente, os que se destacaram foram o atraso na cirurgia anterior $(16,8 \%)$, a inserção de cirurgia de emergência $(14,1 \%)$ e o atraso da equipe cirúrgica $(8,4 \%)$, sendo o sexo feminino o mais frequente $(56 \%)^{22}$, o que corrobora os achados do presente estudo. É importante salientar que essa conduta repercute nos pacientes e em seus familiares, gerando estresse, desconforto, insatisfação com a situação e com a instituição, e na própria instituição, com aumento de gastos e estresse da equipe ${ }^{22,23}$.

O enfermeiro é o responsável pelo planejamento e pelo gerenciamento da rotina do CC e, diariamente, tem de se adaptar às mudanças constantes na programação cirúrgica. Estudo que avaliou as implicações do cancelamento cirúrgico na perspectiva dos enfermeiros mostrou que o profissional se sente responsável, preocupado com os desperdícios decorrentes dos cancelamentos cirúrgicos e com as suas consequências. Embora existam diferenças entre as principais causas da suspensão, em comparação com estudos em outras instituições, observamos que há dados e problemas comuns a todos e que os usuários têm grande prejuízo emocional, financeiro e de saúde ${ }^{22,23}$.

Em uma revisão integrativa, os autores demonstraram que o principal fator gerador de estresse nos pacientes após a suspensão de sua cirurgia foi a falha na comunicação entre o profissional e o paciente. A falta de condições clínicas do paciente foi o principal motivo de cancelamentos e a cirurgia ortopédica foi a especialidade na qual os procedimentos foram mais suspensos ${ }^{24}$. O enfermeiro tem importante função nesse processo, pois, além de prestar assistência ao paciente que irá passar por um procedimento cirúrgico, deve orientar e sanar suas dúvidas para que o paciente sinta segurança ${ }^{24}$. Nesse sentido, a Sistematização da Assistência de Enfermagem Perioperatória (SAEP) é um instrumento de apoio utilizado na avaliação do paciente e de comunicação entre a equipe de enfermagem que garante a continuidade da assistência ${ }^{24}$.

As limitações do estudo envolvem a insuficiência da completude e do correto preenchimento de todos os dados dos procedimentos e das justificativas de cancelamento no prontuário eletrônico do paciente, além do excesso de agendamento e modificações que ocorrem e que dificultaram o andamento da pesquisa e sua conferência.

\section{CONCLUSÃO}

Nesta pesquisa, verificou-se que a taxa de suspensão cirúrgica nos idosos ortopédicos, por motivos extrínsecos, foi de $17,41 \%$, o que corrobora dados de estudos em hospitais universitários. A maioria das cirurgias ocorreu no mês de março, com predomínio do sexo biológico feminino, em pacientes provenientes de fora do município e com idades entre $60 \mathrm{e}$ 69 anos, e a principal categoria de cirurgia foi a fratura de fêmur. Em sua maioria, os procedimentos cirúrgicos foram suspensos apenas uma vez e realizados no período de um a dois dias depois, possuindo como principal motivo de suspensão a extrapolação do tempo previsto da cirurgia anterior, consequentemente ultrapassando o tempo de funcionamento do CC.

A suspensão cirúrgica ainda é um grande desafio aos profissionais e às instituições de saúde. Observou-se grande envolvimento do enfermeiro em gerenciar e minimizar a sua ocorrência, todavia esse não é seu papel exclusivo. É necessário um envolvimento na organização pré-cirúrgica, contando também com membros da equipe cirúrgica. 


\section{REFERÊNCIAS}

1. Oliveira RCS. O processo histórico do estatuto do idoso e a inserção pedagógica na universidade aberta. Rev HISTEDBR [Internet]. 2007 [acessado em 20 maio 2020];(28):278-86. Disponível em: https:// www.fe.unicamp.br/pf-fe/publicacao/5036/art18_28.pdf

2. Gasparotto L, Falsarella G, Coimbra A. As quedas no cenário da velhice: conceitos básicos e atualidades da pesquisa em saúde. Rev Bras Geriatr Gerontol. 2014 [acessado em 20 maio 2020];17(1):201-9. Disponível em: https://doi.org/10.1590/S1809-98232014000100019

3. Monteiro C, Faro A. O cuidador do idoso e sua compreensão sobre a prevenção e o tratamento cirúrgico das fraturas de fêmur. Estud linterdiscip Envelhec [Internet]. 2006 [acessado em 20 maio 2020];10:105-21. Disponível em: https://seer.ufrgs.br/RevEnvelhecer/ article/view/4800/2705

4. Camilo M, Campos L, Viana S, Camargos M, Villa E, Zocrato K. Motivos de cancelamentos, substituição e atrasos de cirurgias eletivas realizadas em um hospital universitário em Minas Gerais. Rev Acred [Internet]. 2017 [acessado em 20 maio 2020];7(13):1-11. Disponível em: https://dialnet.unirioja.es/ descarga/articulo/6130781.pdf

5. Leme L, Sitta M, Toledo M, Henriques S. Orthopedic surgery among the elderly: clinical characteristics. Rev Bras Ortop. 2011;46(3):238-46. https://doi.org/10.1590/S0102-36162011000300002

6. Santos G, Bocchi S. Cancellation of elective surgeries in a Brazilian public hospital: reasons and estimated reduction. Rev Bras Enferm. 2017;70(3):535-5. https://doi.org/10.1590/0034-7167-2016-0084

7. Barbosa MH, Goulart DMM, Andrade EV, Mattia AL. Análisis de la suspensión de cirurgía en um hospital docente. Enferm Glob [Internet]. 2012 [acessdao em 21 abr. 2020]; 11 (26):164-73. Disponível em: http:// dx.doi.org/10.4321/S1695-61412012000200011

8. Gouveia JL, Vilela RP, Ruiz PB, Jericó MC, Ruiz PBO. Cancelamento de cirurgias eletivas: indicador de qualidade em hospital de ensino [Internet]. São José do Rio Preto: Faculdade de Medicina de São José do Rio Preto; 2017 [acessado em 20 maio 2020]. Disponível em: http://sobecc.tmeventos.com.br/anais2017/pdfs/trabalho_2353.pdf

9. Compromisso com a Qualidade Hospitalar. $3^{\circ}$ Caderno de Indicadores CQH [Internet]. São Paulo: Van Moorsel; 2009 [acessado em 20 maio 2020]. 96 p. Disponivel em: http://www.cqh.org.br/portal/pag/doc. php?p_ndoc $=127$

10. Lobo LC, Brito CF, Sabino AS, Almeida MC. Taxa de suspensão de cirurgias no Brasil: uma revisão integrativa. REAC [Internet]. 2019 [acessado em 2 maio 2020];4:e497. Disponível em: https://www. acervomais.com.br/index.php/cientifico/article/view/497

11. Yu K, Xie X, Luo L, Gong R. Contributing factors of elective surgical case cancellation: a retrospective cross-sectional study at a singlesite hospital. BMC Surgery [Internet]. 2017 [acessado em 20 maio 2020];17:100. Disponível em: https://www.ncbi.nlm.nih.gov/pmc/ articles/PMC5594612/pdf/12893_2017_Article_296.pdf https://doi. org/10.1186/s12893-017-0296-9

12. Prin M, Eaton J, Mtalimanja $\mathrm{O}$, Charles $\mathrm{A}$. High elective surgery cancellation rate in malawi primarily due to infrastructural limitations. World J Surg. 2018 [acessado em 2 maio 2020]:42(6):1597-602. Disponível em: https://doi.org/10.1007/s00268-017-4356-1
13. Macedo J, Kano J, Braga E, Ávila MAG, Caldeira SM. Cancelamento de cirurgias em um hospital universitário: causas e tempo de espera para novo procedimento. Rev SOBECC. 2013;18(1):26-34.

14. Pinheiro S, Vasconcelos R, Oliveira J, Matos F, Tonini N, Alves D. Surgical cancellation rate: quality indicator at a public university hospital. REME Rev Min Enferm. 2017 [acessado em 20 maio 2020];21:e1014. Disponivel em: https://doi.org/10.5935/1415-2762.20170024

15. Nascimento L, Tillvitz L, Fonseca L. Suspensão cirúrgica: o ângulo estatístico de um problema de repercussões humanas. Rev Enferm UFPE on line [Internet]. 2013 [acessado em 20 maio 2020];7(Núm. Esp.):6592-600. Disponível em: https://periodicos.ufpe.br/revistas/ revistaenfermagem/article/download/12313/15001 https://doi. org/10.5205/reuol.5058-41233-3-SM.071 1 esp201305

16. Moreira L, Xavier A, Moreira F, Souza L, Araujo O, Santos T, et al. Avaliação os motivos de cancelamento de cirurgias eletivas. Enferm Rev [Internet]. 2016 [acessado em 20 maio 2020];19(2):212-25. Disponível em: http:// periodicos.pucminas.br/index.php/enfermagemrevista/article/view/13156

17. SodréR, Fahl M. Surgeries cancellation in a public hospital at São Paulo city. Rev Adm Saúde [Internet]. 2014 [acessado em 20 maio 2020];16(63):67-70. Available at: https://doi.org/10.5327/Z1519-1672201400630005

18. Soares DS, Mello LM, Silva AS, Martinez EZ, Nunes AA. Fraturas de fêmur em idosos no Brasil: análise espaço-temporal de 2008 a 2012. Cad Saúde Pública. 2014 [acessado em 20 maio 2020];30(12):266978. Disponível em: https://doi.org/10.1590/0102-311×00218113

19. Torri A. Estratégias para minimizar o cancelamento de cirurgias em um hospital escola [Internet]. Florianópolis: Universidade Federal de Santa Catarina; 2019 [acessado em 20 maio 2020]. 117 p. Disponível em: https://repositorio.ufsc.br/bitstream/handle/123456789/198129/ PGCF0100-D.pdf?sequence=-1 \&isAllowed=y

20. Carvalho TA, Sobral CB, Marinho PML, Llapa-Rodriguez EOO, Campos MPA. Suspension of surgery at a university hospital. Rev SOBECC. 2016;21(4):186-91. https://doi.org/10.5327/Z1414-4425201600040002

21. Gouveia Y. Revisão integrativa como estratégia para descrever os principais motivos que levam a suspensão de cirurgias eletivas [Internet]. Uberlândia: Universidade Federal de Uberlândia; 2018 [acessado em 20 maio 2020]. 37 p. Disponívelem: https://repositorio.ufu.br/bitstream/123456789/24236/1/ Revis\%c3\%a3olntegrativaEstrategica.pdf

22. Moraes PGS, Pachêco NMD, Souza e Silva RG, Silva PCV. Clinical and organizational factors related to cancellation of surgical procedures. J Nurs UFPE on line [Internet]. 2017 [acessado em 20 maio 2020];11(7):2645-53. Disponível em: https://periodicos.ufpe. $\mathrm{br} /$ revistas/revistaenfermagem/article/view/23436/19133 https://doi.org/10.5205/reuol.10939-97553-1-RV.1107201701

23. Morgan W, Bernardino E, Wolff LDG. Implications of cancellation of surgery in a surgery department: a descriptive-exploratory study. Online Braz J Nurs [Internet]. 2019 [acessado em 20 maio 2020]; 9(1). Disponível em: http://www.objnursing.uff.br/index.php/nursing/ article/view/j.1676-4285.2010.2591/585

24. Santos AL, Fonseca GG. Impact of suspension of surgery: an integrating review. J Health Connections [Internet]. 2019 [acessado em 20 maio 2020];7(1):46-57. Disponível em: http://revistaadmmade.estacio.br/ index.php/journalhc/article/view/5495/4796583 